\title{
Diagnosis and treatment of postoperative intestinal perforation in infants and young children with congenital heart disease: A report of three cases
}

\author{
YONG-HONG ZHANG, ZHEN-LIANG CHEN, LEI SHI, ZHONG-JIAN CHEN, XIANG-YANG DONG and BO ZHAI
}

Department of Cardiothoracic Surgery, Children's Hospital of Zhengzhou City, Zhengzhou, Henan 450052, P.R. China

Received April 7, 2016; Accepted October 4, 2016

DOI: $10.3892 /$ etm.2018.5963

\begin{abstract}
The aim of the present study was to analyze risk factors of intestinal perforation following surgery for the treatment of congenital heart disease in infants and young children, and to summarize experiences of diagnosis and treatment. A total of 3,270 children, who underwent congenital heart disease surgery under extracorporeal circulation from January 2010 to July 2015, were retrospectively analyzed. Among these children, three $(0.09 \%)$ developed postoperative intestinal perforation. Primary diseases were Tetralogy of Fallot (two cases) and ventricular septal defect combined with atrial septal defect (one case). The age range of the children was 6-11 months and the weight range was $7.3-8.6 \mathrm{~kg}$. Furthermore, these children underwent radical surgery under general anesthesia and extracorporeal circulation in low temperatures. Abdominal symptoms appeared 4-10 days after surgery, and included poor appetite, abdominal distension, intermittent vomiting, high fever, refractory irritability, crying and shortness of breath. One case was confirmed by routine abdominal puncture and the remaining two were confirmed by the detection of free gas under the diaphragm, as revealed by abdominal X-ray. Following the diagnosis of intestinal perforation, emergency intestinal fistula surgery was performed. At 3-5 days post-surgery, the patients underwent treatment by fasting and intravenously administered parenteral nutrition. Diet was increased following recovery of bowel function. All patients recovered following active treatment and 3-4 months following hospital discharge, the fistula was successfully closed. In conclusion, a concerted effort should be made to identify intestinal perforation in infants and young children with postoperative congenital heart disease during emergency surgery. Early diagnosis and treatment may significantly improve prognosis and reduce mortality.
\end{abstract}

Correspondence to: Dr Bo Zhai, Department of Cardiothoracic Surgery, Children's Hospital of Zhengzhou City, 33 Longhu Outer Ring East Road, Zhengdong, Zhengzhou, Henan 450052, P.R. China E-mail: bozhaidoc@163.com

Key words: intestinal perforation, congenital heart disease after surgery, infants, young children, diagnosis, treatment

\section{Introduction}

Cardiac surgery is typically performed under general anesthesia and extracorporeal circulation, and the global incidence of postoperative abdominal complication is $0.12-2 \%$ (1-3). Infants and children with congenital heart disease often suffer from gastrointestinal dysplasia and common postoperative abdominal complications, including peptic ulcer and gastrointestinal bleeding (4). Bowel perforation predominantly occurs on the basis of ulcer and bleeding and its incidence is $0.02-0.08 \%$ (5). However, if treatment is not administered in a time-efficient manner, mortality rates may reach $50 \%$ (6). Symptoms of bowel perforation in the digestive system appear late and do not have clear characteristics. Once symptoms become clear, the disease has progressed; therefore the prognosis of patients is usually poor (7). Usually, perforation is secondary to bleeding and stress ulcers, and the key to treatment is dependent on early diagnosis. Specific diagnostic techniques are also helpful in the diagnosis of perforation and bedside laparoscopy is one such technique that is accurate and minimally invasive. The diagnostic sensitivity of bedside laparoscopy may reach $94 \%$ with timely application, therefore bedside laparoscopy should be administered to patients suspected of having severe abdominal injury (8). Operative indications include gastrointestinal tract necrosis, perforation, acute intestinal obstruction and gastrointestinal bleeding, which conservative treatments have no efficacy against. At present, the advantages of laparoscopy in abdominal exploration and treating abdominal closed injury are pronounced, and laparoscopy has good application prospects as it is minimally invasive, promotes fast recovery in patients (9). In the present study, a total of 3,270 children underwent cardiac surgery under extracorporeal circulation at the Children's Hospital of Zhengzhou City (Zhengzhou, China) between January and July 2010. Among these children, three developed postoperative bowel perforation (0.09\%). Early diagnosis and treatment of intestinal perforation in infants and young children with postoperative congenital heart disease during emergency surgery may significantly improve prognosis and reduce mortality.

\section{Case report}

Clinical data. The primary diseases observed in the three patients (mean age, 8.6 months, 2 female and 1 female) with 
postoperative bowel perforation were Tetralogy of Fallot (two cases) and ventricular septal defect (VSD) plus atrial septal defect (ASD) (one case). These patients underwent radical treatment for Tetralogy of Fallot or repair treatment for VSD plus ASD under general anesthesia and hypothermic extracorporeal circulation, respectively.Patients whounderwent radical treatment for Tetralogy of Fallot were provided with milk through the nasal passage from postoperative day two. However, in one of the patients with Tetralogy of Fallot, sudden cardiac rate drops occurred on postoperative day four, with the lowest recorded cardiac rate being $\sim 60 \mathrm{bpm}$ and the lowest recorded blood pressure being $40 / 21 \mathrm{mmHg}$. Following 3 min cardiopulmonary resuscitation and adrenaline administration, sinus rhythm was restored and blood pressure began to increase. At postoperative day six, the patients were successfully disconnected from artificial respirators and trachea cannulas were removed. At postoperative day eight, the patients began to ingest a liquid diet and were moved to the common ward. At postoperative day nine, the patients experienced anorexia, abdominal distension and hyperpyrexia, which peaked at $39.8^{\circ} \mathrm{C}$. Fasting, rehydration, cleansing enema and gastrointestinal decompression were administered unsuccessfully. Abdominal puncture was subsequently performed and yellow intestinal fluid was obtained, suggesting bowel perforation. The third patient, with VSD plus ASD, exhibited the following symptoms: Poor appetite, abdominal distension, irritability and crying on the first and second day following the ingestion of solid foods. Abdominal distension was not markedly relieved following gastrointestinal decompression. Furthermore, abdominal X-ray examination revealed free gas in the lower diaphragm (Fig. 1A and B), suggesting that bowel perforation had occurred. In the three patients, the interval between the appearance of abdominal symptoms and the diagnosis of intestinal perforation and intestinal fistulation surgery was 1-2 days. Intervals were from the onset of symptoms to the diagnosis of intestinal perforation and these intervals were all $>24 \mathrm{~h}$, which in the opinion of the current authors, was too long. Furthermore, bowel edema and adhesion were severe; therefore, intestinal fistulation surgery was selected for the patients instead of one-stage anastomosis following bowel resection. Fasting and rehydration treatment during parenteral nutrition were performed 3-5 days following removal of the trachea cannula, when patients were in a stable condition. Bowel function of the patients was restored at 5 days following fasting and hydration, and a liquid diet was gradually administered. The duration of postoperative hospital stays following fistulation was 15-26 days. At 3-4 months following recovery and discharge, patients underwent fistula-closing surgery. One week post-surgery, all patients were discharged from hospital and had normal diet. Within 1 week of leaving hospital, patients were fed with milk powder, $80-120 \mathrm{ml} / \mathrm{kg}$ a day, once every $3 \mathrm{~h}$. One Week later, follow up was conducted by phone, concerning the patients' diets, stools and weights. All 3 patients exhibited normal eating and bowel habits and experienced a weight increase of $0.5-1 \mathrm{~kg}$. No adverse effects were recorded when patients returned to the hospital for check-ups at 1, 3 and 12 months.

Surgical methods. Under general anesthesia using propofol injection (4-12 mg/kg/h; Corden Pharma International,
Plankstadt, Germany), sufentanil citrate injection (3 $\mu \mathrm{g} / \mathrm{kg} / \mathrm{h}$, Yichang Renfu Pharmaceutical Co., Ltd., Yichang, China), dexmedetomidine hydrochloride injection $(0.5 \mu \mathrm{g} / \mathrm{kg} / \mathrm{h}$, Jiangsu hengrui medicine Co., Ltd., Lianyungang, China) and vecuronium bromide (administered via intravenous injection; maintenance dose 0.02-0.03 mg/kg.h; Xiangbei Welman Pharmaceutical, Co., Ltd; tracheal intubation measurement, $0.08-0.1 \mathrm{mg} / \mathrm{kg}$ ), and extracorporeal circulation at low temperatures, radical surgery was performed through an incision in the sternum midline on the two patients with Tetralogy of Fallot. Aorta blocking durations were 63 and $102 \mathrm{~min}$, and extracorporeal circulation times were 94 and $141 \mathrm{~min}$ respectively. Under the same general anesthesia protocol as above and extracorporeal circulation, the patient with VSD plus ASD underwent repair surgery via right subaxillary incision. Aorta blocking and extracorporeal circulation times were 68 and $112 \mathrm{~min}$, respectively. An exploratory laparotomy was performed following the postoperative development of abdominal symptoms, and the results suggested intestinal perforation. A $5.0 \mathrm{~cm}$ incision at the left rectus abdominis was performed in one patient with Tetralogy of Fallot. When the abdominal cavity was opened, a large amount of yellow cloudy purulent fluid was expunged. Further inspection of the abdominal cavity revealed congestion in the whole small intestine and partial congestion in the colon, which appeared to be brittle. Pus was present on the surfaces, which was removed, and the small intestinal adhesion was separated. Two ulcer perforations were present in the ileum $40 \mathrm{~cm}$ from the ileocecus (Fig. 1C), which were $\sim 1.5 \times 1.5 \mathrm{~cm}$. Following intestinal decompression, the ileum was removed at the perforation site and double-cavity ileal fistula surgery was performed through the left side of the abdominal incision.

A left rectus incision was also performed on the other patient with Tetralogy of Fallot, with the perforated area $\sim 20 \mathrm{~cm}$ from the ileocecus. Due to significant intestinal edema and congestion, single-cavity fistulation surgery at the ileum was performed. For the patient who underwent VSD plus ASD repair, five perforations were identified in the terminal ileum $\sim 10 \mathrm{~cm}$ from the ileocecum. The largest perforation was $1 \mathrm{~cm}$ in diameter (Fig. 1D), and the smallest was $0.3 \mathrm{~cm}$ in diameter. Viscous yellow-green feces were also observed in the perforated area. Following intestinal inspection, a seromuscular ulcer was identified in the jejunal canal. Following the separation of intestinal adhesion, $\sim 5 \mathrm{~cm}$ of diseased bowel was excised. The intestinal canal at the distal end of the incision was embedded in the abdominal cavity, whereas the intestinal canal at the proximal end of the incision was retrieved and placed at the lower edge of the rectus incision for fistulation (Fig. 1E). The excised diseased bowel was sent for subsequent pathological examination. Staining was performed using hemotoxylin and eosin and analyzed using a light microscope (magnification, x10, Fig. 1F).

Intestinal fistula surgeries were successfully completed in all three patients. Fasting and rehydration treatment during parenteral nutrition was performed 3-5 days following removal of the trachea cannula, and when patients were in a stable condition. Bowel function was restored in patients following 3-5 days of treatment and a liquid diet was gradually administered. Postoperative hospital stays following fistulation ranged from 15 to 26 days. At 3-4 months following recovery and 

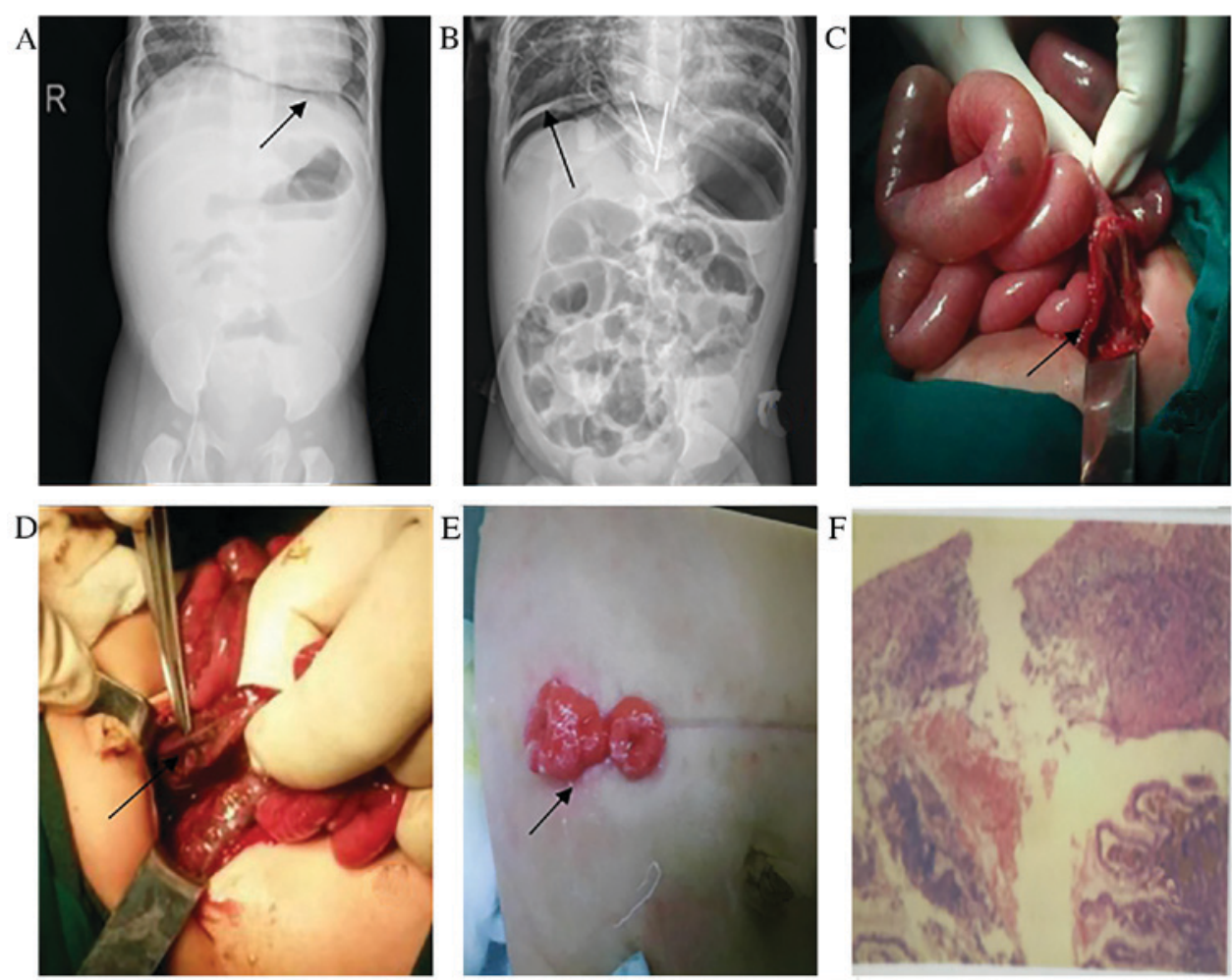

Figure 1. Images of intestinal perforation following surgery for congenital heart disease. (A) X-ray image of abdomen following surgery in a patient with Tetralogy of Fallot. Arrow indicates subdiaphragmatic free air. (B) X-ray image of abdomen following ventricular septal defect repair. Arrow indicates subdiaphragmatic free air. (C) Intestinal perforation post-surgery in a child with Tetralogy of Fallot. (D) Intestinal perforation following ventricular septal defect repair. (E) Fistulation following intestinal perforation. (F) Pathology results of an intestinal segment excision for intestinal perforation. Staining, hemotoxylin and eosin, magnification, $\mathrm{x} 10$.

discharge, the patients underwent fistula-closing surgery and were discharged from the hospital one week post-surgery. Patients were monitored up for 3-4 years following discharge. During monitoring, diet, bowel movements, and weights of patients were compared with peers of the same age and were deemed to be normal.

\section{Discussion}

Postoperative abdominal complications are closely associated with the effect of extracorporeal circulation on gastrointestinal tract blood flow in congenital cardiac disease, and in infant bowel development (10). The development of the intestinal lamina muscularis mucosae is poor in infants and the mesentery is soft and long. Thin intestinal walls with a high permeability and poor barrier function may result in an imperfect oral tolerance enabling intestinal endotoxins, incomplete digestion products and other allergens to enter the body through the intestinal mucosa. These allergens may induce systemic infection and allergic diseases. It has previously been reported that intestinal mucosal blood flow begins to decline at the beginning of extracorporeal circulation, and that blood flow decreases by $50 \%$ in 30 min (11). These physical characteristics of infant bowels, when combined with reduced intestinal blood flow during extracorporeal circulation and postoperative low cardiac output syndrome or mesenteric vascular contraction induced by a sudden increase in blood pressure, are risk factors for gastrointestinal complications $(12,13)$. The cause of postoperative bowel perforation in patients with congenital cardiac disease is gastrointestinal ischemic anoxic injury, due to low cardiac output syndrome (14). Among the patients evaluated in the present study, those with Tetralogy of Fallot had been exhibiting hypoxia for a long period of time, which affected the growth of the gastrointestinal tract. As the durations of aortic blocking and extracorporeal circulation were prolonged during surgery, patients developed postoperative bowel ischemia induced by low cardiac output syndrome at different levels for a long period of time.

Typical postoperative abdominal complications in patients with congenital cardiac disease include: Changes in the colour of the gastric fluid, abdominal distension, fever, abdominal pain and black stools (15). Following the detection of these symptoms, measures such as cardiac therapy and diuresis to improve heart function and the intestinal microcirculation environment should be actively performed in patients. At the same time, treatments including fasting, rehydration, cleansing enema, gastrointestinal decompression and the administration of anti-acid drugs should be performed (8). If treatment is not provided in a time-efficient manner, this may lead to bowel perforation, sepsis and shock (16). Complications arising from bowel perforation, such as sepsis and shock, may increase the incidence of postoperative heart failure, respiratory failure and multiple organ function failure in children; as such, these complications require early diagnosis and treatment. Typically, when symptoms manifest, these complications progress to the middle or later stages resulting in a poor prognosis $(17,18)$. For children who undergo surgery for congenital cardiac diseases, if symptoms including abdominal distension, crying, vomiting, 
black stool and sustained high fever appear following eating, treatment options such as fasting, gastrointestinal decompression and cleansing enema should be actively provided once heart and lung factors have been excluded and the stability of respiratory circulation ensured. If symptoms are not relieved following treatment, the occurrence of gastrointestinal perforation should be highly suspected.

In the present study, the trachea cannula was removed from patients when their respiratory circulation became stable. However, abdominal distention, fever, vomiting and other symptoms appeared 1-2 days following their return to a solid diet. Furthermore, abdominal distention was not significantly relieved following treatment with fasting, rehydration, gastrointestinal decompression and cleansing enema in these patients. Bowel perforation was confirmed by abdominal X-ray (19) and abdominal puncture examinations, and intestinal fistula (20) surgery was subsequently performed. Three fistulation surgeries were performed by general physicians. Although bowel perforation was confirmed within 1-2 days, it was detected during the surgery that bowel adhesion and edema were severe. Therefore, to prevent the occurrence of perforation, intestinal fistulation treatment was performed rather than one-stage anastomosis at the perforation site following resection of the bowel.

For infantile patients with bowel perforation, fasting and parenteral nutrition therapy should be applied following fistulation treatment as comprehensive treatment has been previously demonstrated to have a significant curative effect (21). For children who develop bowel perforation following surgery for congenital cardiac disease, the intake of liquid should be controlled in accordance with baseline requirements to reduce the load on the heart (22). Cardiac therapy, diuresis, myocardial nutrition and anti-infection therapy should also be provided. These measures should ensure that the intake and discharge balance of water is maintained. However, the acid-base balance, and electrolyte and protein levels should be reviewed periodically to efficiently provide corrective treatment. Three to four months following patient recovery and discharge, fistula-closing surgery should be performed according to the physical condition of the patient (23).

In conclusion, postoperative low cardiac output syndrome may induce gastrointestinal tract ischemia, intestinal digestion and absorption dysfunction after eating, and increase abdominal pressure. These are the main factors that lead to intestinal perforation following surgery for congenital cardiac disease in infant patients. Bowel development characteristics in infants and young children, and the effects of heart disease on intestinal canal development prior to surgery influence the occurrence of perforation (5). For such high-risk children, appropriate measures should be taken to reduce the occurrence of postoperative low cardiac output syndrome $(24,25)$, and ensure normal blood pressure and heart rate. For children exhibiting brown gastric fluid and black stools, or milk retention following nasogastric feeding, treatment with fasting, rehydration, $\mathrm{H} 2$ receptor antagonists and proton pump inhibitors have lead to positive therapeutic outcomes in the majority of cases $(26,27)$. If symptoms including abdominal distension, repeated high fever, lethargy and irritability are exhibited, abdominal X-ray examination should be performed. In cases where intestinal perforation is suspected, exploratory laparotomy should be performed to avoid infectious shock induced by the diffusion and absorption of intestinal endotoxins, which may increase the risk of mortality in children (28). For children with a confirmed diagnosis of intestinal perforation due to the presence of congestion and edema close to perforation sites, fistulation surgery should be performed $(29,30)$. This may reduce the duration of surgery and surgical trauma, and is conducive to the absorption of congestion and edema.

\section{References}

1. Hessel E II: Abdominal organ injury after cardiac surgery. Semin Cardiothorac Vasc Anesth 8: 243-263, 2004.

2. Huddy SP, Joyce WP and Pepper JR: Gastrointestinal complications in 4473 patients who underwent cardiopulmonary bypass surgery. Br J Surg 78: 293-296, 1991.

3. Mierdl S, Meininger D, Dogan S, Aybek T, Wimmer-Greinecker G, Lischke V and Kessler P: Abdominal complications after cardiac surgery. Ann Acad Med Singapore 30: 245-249, 2001.

4. Christenson JT, Schmuziger M, Maurice J, Simonet F and Velebit V: Postoperative visceral hypotension the common cause for gastrointestinal complications after cardiac surgery. Thorac Cardiovasc Surg 42: 152-157, 1994.

5. Viana FF, Chen Y, Almeida AA, Baxter HD, Cochrane AD and Smith JA: Gastrointestinal complications after cardiac surgery: 10 year experience of a single Austrilian centre. ANZJ Surg 83: 651-656, 2013.

6. Christenson JT, Aeberhard JM, Badel P, Pepcak F, Maurice J, Simonet F, Velebit V and Schmuziger M: Adult respiratory distress syndrome after cardiac surgery. Cardiovasc Surg 4: 15-21, 1996.

7. Viana FF, Chen Y, Ahneida AA, Baxter HD, Cochrane AD and Smith JA: Gastrointestinal complications after cardiac surgery: 10 year experience of a single Austrilian centre. ANZ J Surg 83: 651-656, 2013.

8. Hackert T, Kienle P, Weitz J, Werner J, Szabo G, Hagl S, Büchler MW and Schmidt J: Accuracy of diagnostic laparoscopy for early diagnosis of abdominal complications after cardiac surgery. Surg Endosc 17: 1671-1674, 2003.

9. Wei JB, Liang WQ and Xie GS: The application of laparoscopic technique in diagnosis and treatment of abdominal injury: With a report of 102 cases. J Laparoscop Surg 16: 468-470, 2011.

10. Sever K, Ozbek C, Goktas B, Bas S, Ugurlucan M, Mansuroglu D: Gastrointestinal complications after open heart surgery: Incidence and determinants of risk factors. Angiology 65: 425-429, 2014.

11. Sack FU, Reidenboch B, Dollner R, Schledt A, Gebhard MM and Hagl S: Influence of steroids on microvascular perfusion injury of the bowel induced by extracorporeal circulation. Ann Thorac Surg 72: 1321-1326, 2001.

12. Sorokina EA, Morova NA, Tsekhanovich VN, Kopeǐkin SA, Mil'chenko MV and Malkov AV: Stress-dependent lesions of the gastroduodenal mucosa during operations with cardiopulmonary bypass. Kiln Med (Mosk) 85: 51-55, 2007 (In Russian).

13. Stazka J, Jaguś K, Krawczyk E, Matuszek M and Rudzki S: Abdominal complications after cardiac surgery in cardiopulmonary bypass. Ann Univ Mariae Curie Sklodowska Med 59: 387-391, 2004

14. Rodriguez R, Robich MP, Plate JF, Trooskin SZ and Sellke FW: Gastrointestinal complications following cardiac surgery: A comprehensive review. J Card Surg 25: 188-197, 2010.

15. Zacharias A, Schwann TA, Parenteau GL, Riordan CJ, Durham SJ, Engoren M, Fenn-Buderer N and Habib RH: Predictors of gastrointestinaI compIications in cardiac surgery. Tex Heart Inst J 27: 93-99, 2000.

16. Heikkinen LO and Ala-Kulju KV: Abdominal complications following cardiopulmonary bypass in open-heart surgery. Scand J Thorac Cardiovasc Surg 21: 1-7, 1987.

17. Zhou H, Sun N, Zhang X, Xie H, Ma L, Shen Z, Zhou X and Tao T: Transumbilical laparoendoscopic single-site pyeloplasty in infants and children: Initial experience and short-term outcome. Pediatr Surg Int 28: 321-325, 2012.

18. Kargl S, Maier R, Gitter R and Pumberger W: Necrotizing enterocolitis after open cardiac surgery for congenital heart defects-a serious threat. Klin Padiatr 225: 24-28, 2013.

19. Cheng DS, Kong LM, Hu QM, Ma WP and Ding Y: Clinical discussion on neonatal intestinal perforation. Chin Pediat Emerg Med: 62-63, 2005 (In Chinese). 
20. Xue G, Tian FZ, Wang PH, Zhang GH, Cao YK, Zhang L, Gong JQ and Gong J: Clinical features of spontaneous perforation of the colon. Chin J Digest Surg 4: 312, 2010 (in Chinese).

21. Liu ZR and Xu XR: Total parenteral nutrition in the treatment of bowel perforation in children. Chin J Pediatr Surg: 210-212, 2001 (in Chinese).

22. Cui YL, Song JB, Yin HZ, et al: Management of body fluid balance and electrolyte after open heart surgery in children. Chinese Gen Prac 11: 1038-1039, 2008 (in Chinese).

23. Lu Q, Zhou XY and Liu ZY: Enterostomy closure: How to select an opportunity after enterostomy in children. Zhongguo Dang Dai Er Ke Za Zhi 14: 69-70, 2012 (In Chinese).

24. Xu F and Chen X: The treatment of rhubarb on stress ulcer after cardiopulmonary bypass in children. Chin Pediatr Emerg Med: 359, 2011 (in Chinese).

25. Dong G, Liu C, Xu B, Jing H, Li D and Wu H: Postoperative abdominal complications after cardiopulmonary bypass. J Cardiothorac Surg 7: 108, 2012.
26. González Ojeda A, Orozco Mosqueda A, Barrera Zepeda L, Fuentes Orozco C, Avalos González J, Hinojosa Alarcón F, Paredes Carlo CO and Victal Adame O: Abdominal complications after cardiopulmonary procedures. Rev Gastroenterol Mex 64: 61-69, 1999 (In Spanish).

27. Simić O, Strathausen S, Hess W and Ostermeyer J: Incidence and prognosis of abdominal complications after cardiopulmonary bypass. Cardiovasc Surg 7: 419-424, 1999.

28. Gao P, Zhang XW, Liu XL, Wang H, Liang YF, Sun JX, Wang DH and Li HN: A case of intestinal perforation correction of double outlets in children's right ventricular. Chin J Pediatr Surg: 557-558, 2014 (in Chinese).

29. Cai SL, Chen T, Yao LQ and Zhong YS: Management of iatrogenic colorectal perforation: From surgery to endoscopy. World J Gastrointest Endosc 7: 819-823, 2015.

30. Putcha RV and Burdick JS: Management of iatrogenic perforation. Gastroenterol Clin North Am 32: 1289-1309, 2003. 